\title{
Uma Experiência em Sala de Aula usando um Livro Didático convertido para um Livro Aumentado
}

\author{
Adilson Vahldick ${ }^{1}$, Douglas Linhares Bittencourt ${ }^{1}$ \\ ${ }^{1}$ Departamento de Engenharia de Software, Centro de Educação Superior do Alto Vale \\ do Itajaí, Universidade do Estado de Santa Catarina (Udesc) - Ibirama, SC - Brasil \\ adilson.vahldick@udesc.br, douglaslbittencourt@gmail.com
}

\begin{abstract}
Textbooks are an expensive investment for the government, on the other hand, students are big consumers of digital material. Mobile devices became popular in the hands of these young people, offering an opportunity to incorporate new utilities related to their learning. In this work, an experiment was conducted with a ninth-grade geography class in which they used a textbook, already adopted by them, integrated with an augmented reality application, transforming it into an enlarged book. In this application were developed maps of the African continent in $3 D$ that were visualized when pointed to its printed version in the book. Pre-and post-tests were applied to verify if there was any improvement in African knowledge. In addition, they completed a questionnaire with six questions to discuss their application and experience. The results showed a statistically significant improvement of $14 \%$ in the mean of the score between the tests. Students demonstrated satisfaction and would like to have more experiments of this kind. These results present a possibility of government investment in developing augmented reality applications reusing the investment already made with textbooks.
\end{abstract}

Resumo. Livros didáticos são um investimento caro para o governo, e na contramão, os estudantes são grandes consumidores de material digital. Os dispositivos móveis se popularizaram nas mãos desses jovens, oferecendo uma oportunidade para incorporar novas utilidades relacionadas ao seu aprendizado. Neste trabalho, foi conduzida uma experiência com uma turma de geografia do nono ano em que utilizaram um livro didático, já adotado por eles, integrado a uma aplicação de realidade aumentada, transformando-o num livro aumentado. Nessa aplicação foram desenvolvidos mapas do continente africano em $3 D$ que eram visualizados quando apontados para sua versão impressa no livro. Foram aplicados pré e pós testes para verificar se houve alguma melhoria no desempenho sobre o conhecimento do continente africano. Além disso, eles completaram um questionário com seis questões para opinarem sobre a aplicação e a experiência. Os resultados demonstraram uma melhora estatisticamente significante em 14\% na média da nota entre os testes. Os estudantes demonstraram satisfação e gostariam que houvessem mais experiências desse gênero. Esses resultados apresentam uma possibilidade de investimento do governo em desenvolver aplicações de realidade aumentada reaproveitando o investimento já realizado com os livros didáticos. 
VIII Congresso Brasileiro de Informática na Educação (CBIE 2019)

Anais do XXX Simpósio Brasileiro de Informática na Educação (SBIE 2019)

\section{Introdução}

As tecnologias que vem dando suporte às tarefas de ensino e aprendizado permitem grande interatividade entre os estudantes e o conteúdo que estão estudando. Apesar de tudo, o material didático impresso continua com as suas vantagens em prover um meio sistemático de estudar. Entretanto, livros didáticos no Brasil não são inteiramente seguidos pelos professores pela questão de adaptá-los às suas realidades regionais e convicções pedagógicas (NETO; FRACALANZA, 2003). Neste contexto, uma possibilidade é suportar as decisões do professor provendo material suplementar que pode ser integrado aos livros didáticos, e assim, auxiliando o professor na preparação de suas aulas.

A Realidade Aumentada (RA) é uma tecnologia que integra em tempo real a informação digital processada por um computador com informações vindas do mundo real por meio de adequadas interfaces de computador (AMIN; GOVILKAR, 2015). A sobreposição do mundo virtual no real, ou seja, o conteúdo gerado por computador é adicionado ou incorporado na experiência do mundo real, para que ambos possam ser experimentados juntos (MANN et al., 2018).

A sua aplicação na educação permite a conexão entre os domínios físicos e digitais, oferecendo uma experiência em que se combinem a informação real e virtual, auxiliando os estudantes a entenderem problemas abstratos ao visualizarem objetos "reais" (BILLINGHURST; DÜNSER, 2012; CUENDET et al., 2013). Livros aumentados assemelham-se com os livros impressos, mas eles possuem um conteúdo virtual e cenas animadas que complementam o conteúdo impresso (BILLINGHURST; DÜNSER, 2012). Os estudantes podem mudar suas perspectivas ou direções movendo a cabeça ou o livro porque o conteúdo $3 \mathrm{D}$ está ligado à página do livro (YUEN; YAOYUNEYONG; JOHNSON, 2011), permitindo experiências mais imersivas e situadas (BOWER et al., 2014).

Esse artigo apresenta os resultados de uma experiência para verificar a validade no desenvolvimento de pequenas aplicações em dispositivos móveis para visualizar objetos $3 \mathrm{D}$ a partir de mapas em livros didáticos usados pelos estudantes, se tornando uma alternativa para aumentar o valor no uso desses livros.

O trabalho está estruturado da seguinte maneira: na seção 2 são apresentadas as aplicações da realidade aumentada na educação e os livros aumentados. A seção 3 explana o desenvolvimento da aplicativo. A seção 4 detalha o experimento e a quinta apresenta os resultados. As conclusões são apresentadas na sexta seção.

\section{Realidade Aumentada na Educação e Livros Aumentados}

Educadores e pesquisadores investem em novas tecnologias para apoiar o processo de ensino-aprendizagem. Uma dessas tecnologias que tem despertado interesse na última década é a Realidade Aumentada (RA), que permite ao usuário visualizar e manipular objetos 3D virtuais em um ambiente real (HORNECKER; DÜNSER, 2007). A RA tem um forte potencial para experiências de aprendizagem, pois ela permite a exploração e descoberta da natureza virtual conectada da informação no mundo real (JOHNSON et al., 2010). A RA não só permite uma imersão natural e motivadora ao aluno, como também faz com que aumente sua percepção, proporcionando melhor aprendizado e 
VIII Congresso Brasileiro de Informática na Educação (CBIE 2019)

Anais do XXX Simpósio Brasileiro de Informática na Educação (SBIE 2019)

uma maior compreensão do conteúdo didático apresentado, sem a necessidade de aquisição do objeto de estudo (CARDOSO et al., 2014).

A RA é a amplificação da percepção sensorial por meio de recursos computacionais. Assim, associando dados computacionais ao mundo real, a RA permite uma interface mais natural com dados e imagens geradas por computador (CARDOSO et al., 2007). A RA depende do hardware usado para aumentar o conforto e a imersão. Dispositivos móveis são mais confortáveis que head-mounted displays (HMD) quando se trata de usos mais prolongados (ASAI; KOBAYASHI; KONDO, 2005).

O livro aumentado é um sistema que aumenta os elementos multimídia em um livro para trazer efeitos educacionais adicionais ou diversão (YANG et al., 2008). Usuários sem conhecimentos avançados em informática conseguem usar esses livros aumentados (GAZCÓN; LARREGUI; CASTRO, 2016). Estes livros possuem vários marcadores ancorados nas suas páginas em que um dispositivo os detecta e exibe os elementos virtuais (IVANOVA; ALIEV; IVANOV, 2014). Mas, para isso, os dispositivos precisam de modelos 3D (cenas e conteúdos relacionados aos marcadores) previamente instalados. A interação inicia quando o usuário aponta a câmera para os marcadores no livro. Um marcador baseado em RA usa imagens detectadas por uma câmera. Os marcadores são utilizados para fazer o posicionamento dos elementos virtuais no mundo real. Eles são reconhecidos com base nas características da imagem. Para isso, devem ser usadas imagens com um bom nível de contraste e devem ter características distribuídas uniformemente, mas evitando padrões repetidos, bordas arredondadas e imagens borradas (SCHMITZ; SOLANO; LOPES, 2017).

Os livros aumentados provaram ser válidos para efetivamente aumentar a eficácia do aprendizado (CHEN; ZHANG, 2008; GOMES; GOMES; OLIVEIRA, 2017). Para o ensino básico existem exemplos de observação astronômica e ambientes biológicos (CHEN; ZHANG, 2008), educação visual (GOMES; GOMES; OLIVEIRA, 2017), história (GRASSET; DÜNSER; BILLINGHURST, 2008) e geometria (KIRNER; REIS; KIRNER, 2012). Para educação superior, existem exemplos de habilidades de aprendizagem espacial em cursos de engenharia (MARTÍN-GUTIÉRREZ; CONTERO; ALCAÑIZ, 2010), engenharia mecânica (GUTIÉRREZ; ASME, 2011), ferramentas de corte (IVANOVA; ALIEV; IVANOV, 2014) e paleontologia (GAZCÓN; LARREGUI; CASTRO, 2016).

\section{Desenvolvimento}

O objetivo do aplicativo de RA, descrito nesse trabalho, é prover aos estudantes uma diferente visão do livro didático de geografia deles adicionando modelos 3D. Através de uma entrevista com a professora de geografia do nono ano da Escola Municipal Christa Sedlacek pôde-se definir o período para fazer o experimento e por consequência, o assunto a ser desenvolvido no aplicativo. Com base no livro de Adams e Adams (2016), a professora determinou a "Unidade 7 - África: heranças, conflitos e diversidades" a ser complementada com o aplicativo de RA. Os modelos 3D foram desenvolvidos com base nos mapas de clima, relevo e vegetação, conforme a Figura 1. 


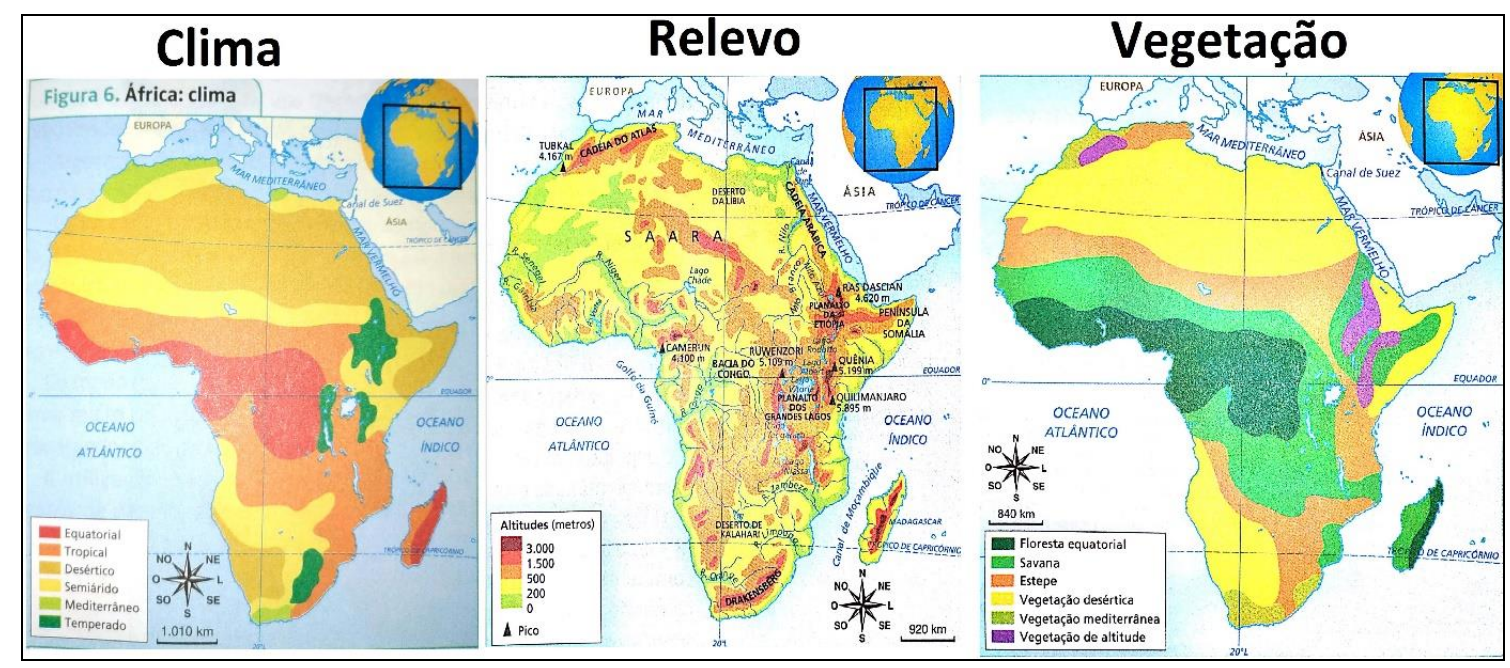

Figura 1 - Marcadores utilizados no aplicativo

O aplicativo foi implementado em Unity ${ }^{1}$ usando Vuforia $\mathrm{SDK}^{2}$ para os recursos de RA. Os requisitos mínimos para executar o aplicativo são: Android com OS 4.1 ou posterior, CPU ARMv7 com suporte NEON ou CPU Atom, OpenGL ES 2.0 ou posterior (UNITY, 2019a). Os mapas da Figura 1 foram utilizados como marcadores. Esses mapas foram modelados em 3D usando Blender ${ }^{3}$. Os demais itens usados na modelagem foram adquiridos na loja da Unity.

A Figura 2 apresenta o resultado da modelagem de cada um dos três mapas respectivamente. Com base no modelo plano dos mapas, através do Blender, foram acrescentados os relevos e a coloração. Existem elementos sugeridos pela professora que foram inseridos nos modelos para reforçar a associação do conteúdo com a localização no mapa. Por exemplo, no modelo da Figura $2 \mathrm{a}$ - sobre climas - existem camelos e escorpiões na região desértica e nuvens e florestas mais densas nas regiões equatoriais. No modelo da Figura $2 b$ - sobre relevos - foram adicionados os nomes das principais cadeias de montanhas. Por diversão, no modelo da Figura 2c, foram adicionados alguns personagens dos filmes de Madagascar da Dreamworks.

Através do aplicativo, quando o estudante apontar a câmera no livro para uma das imagens da Figura 1, o aplicativo reconhecerá a marcação da imagem alvo e mostrará o respectivo modelo $3 \mathrm{D}$. O estudante pode interagir e explorar esse modelo usando os dois dedos para rotacionar nos três eixos, aumentar ou diminuir o zoom.

\footnotetext{
1 A Unity é uma plataforma de desenvolvimento de jogos em 2D e 3D. Seu motor de jogos permite desenvolver para diversas plataformas como PCs, consoles, web e HMD (UNITY, 2019b).

${ }^{2}$ Vuforia é um SDK para a construção de aplicativos de RA para diversos dispositivos e plataformas, como: tablets, smarthphones, notebooks, óculos de RA e capacetes de Realidade Virtual (PTC, 2019).

3 Blender é uma ferramenta de criação 3D gratuita de código aberto, possibilitando a criação de modelagem, manipulação, animação, simulação, renderização, composição e rastreamento de movimento, até mesmo edição de vídeo e criação de jogos (BLENDER, 2019).
} 
VIII Congresso Brasileiro de Informática na Educação (CBIE 2019)

Anais do XXX Simpósio Brasileiro de Informática na Educação (SBIE 2019)

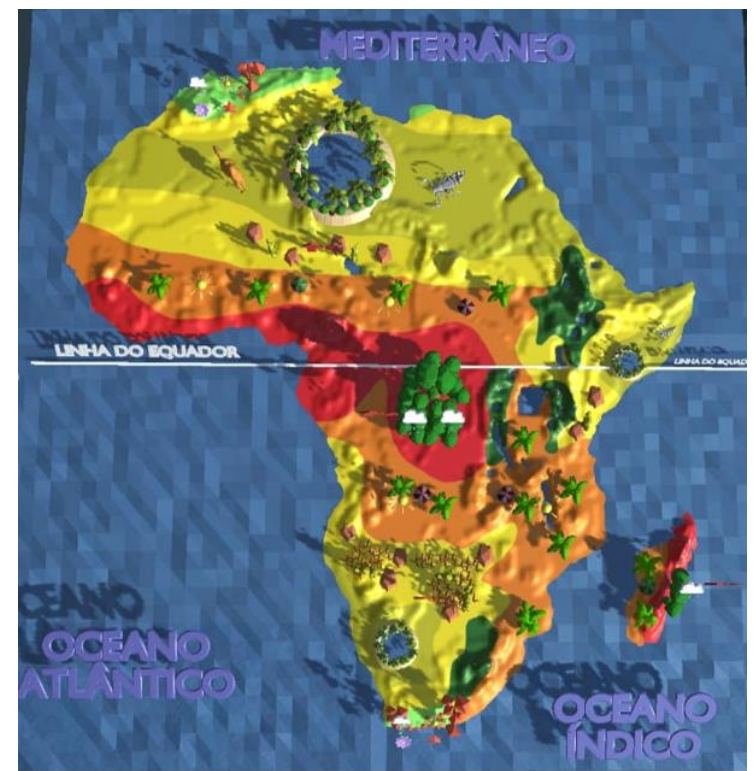

(a)

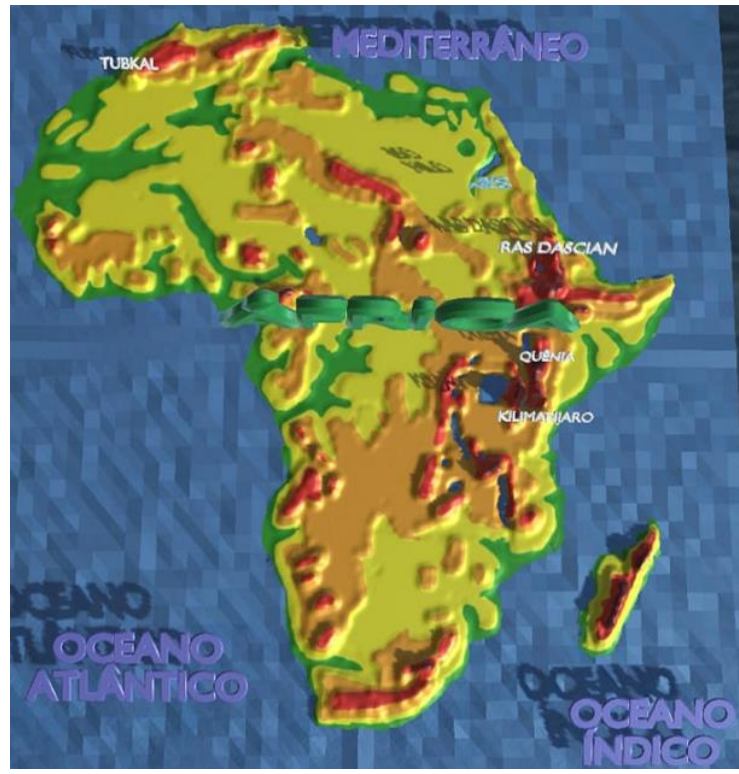

(b)

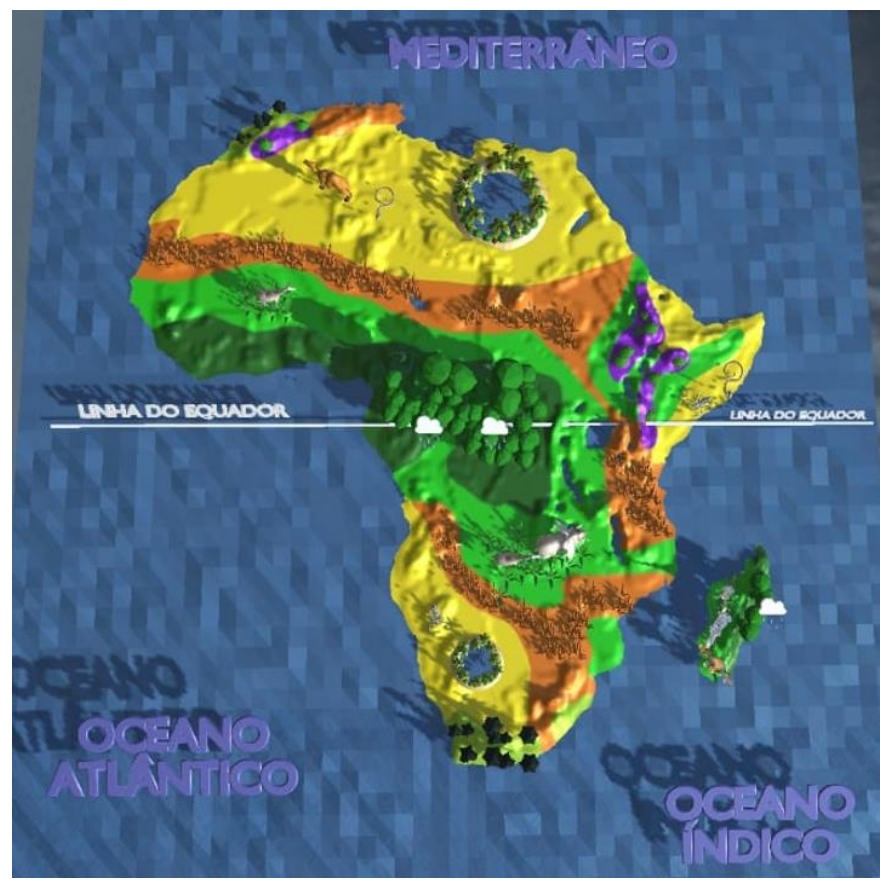

(c)

Figura 2 - Modelos 3D do Mapa de (a) Clima, (b) Relevo e (c) Vegetação

\section{Metodologia}

O esquema da Figura 3 apresenta o processo da experimentação com o aplicativo. Após a professora conduzir as suas aulas pelo método tradicional com o assunto da "Unidade 7-África" do livro de Adams e Adams (2016), o estudo foi conduzido em uma sessão de 60 minutos com 28 alunos presentes do nono ano (idades entre 14 e 15 anos). No início, os estudantes responderam um pré-teste aplicado com um questionário de 10 questões 
VIII Congresso Brasileiro de Informática na Educação (CBIE 2019)

Anais do XXX Simpósio Brasileiro de Informática na Educação (SBIE 2019)

de múltipla escolha. Em seguida, os alunos puderam usar seus celulares (normalmente é proibido seu uso nas aulas) para baixar e instalar o aplicativo de RA. Porém, nem todos tinham seus celulares consigo. Os pesquisadores e a professora emprestaram os seus celulares, além de disponibilizarem um tablet.

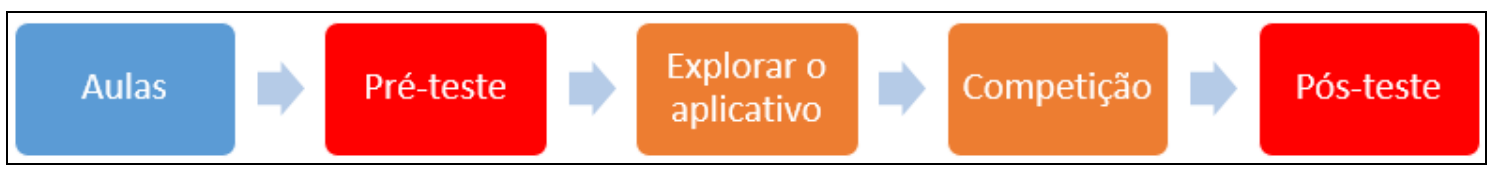

Figura 3 - Estratégia de Condução do Experimento

Os estudantes tiveram 10 minutos para usar o aplicativo explorando os três mapas. Foi sugerido a eles verificarem onde estão localizados cada tipo de relevo, clima e vegetação, e os elementos inseridos em cada um dos tipos.

Baseado em Regueras et al. (2009) que apontam que alguns estudantes são mais motivados em realizar tarefas quando competem, objetivando promover a aprendizagem e reforçar o valor do aplicativo, foi organizada uma competição entre times formados pelos próprios estudantes. Foram constituídos 7 times e eleitos um representante de cada time. A competição era baseada em uma pergunta lançada pelos investigadores e o representante do time que levantasse primeiro o braço tinha 5 segundos para responder. Em caso de acerto, a equipe ganhava 1 ponto. Quando erravam, a equipe perdia 1 ponto, e outro time na sequência podia responder. A competição foi conduzida com 15 questões que exigiam o acesso do aplicativo para serem respondidas. Por exemplo, "No mapa da vegetação, quantos oásis existiam no deserto?", ou "Qual o nome do pico mais ao nordeste da África?".

Após a competição foi aplicado um pós-teste contendo as mesmas questões do pré-teste, porém as questões e as suas respostas estavam em outra sequência. Além disso, eles também responderam outro formulário com seis questões (quatro objetivas e duas abertas) para opinarem sobre o aplicativo e a experiência.

\section{Resultados}

\subsection{Pré-teste e Pós-teste}

De 28 participantes, tanto no pré-teste quanto no pós-teste, 23 deles alcançaram a nota 7,0 (nota de aprovação) ou superior. A média foi melhorada em 14\%. A Tabela 1 apresenta a comparação entre os testes. A Figura 4 apresenta a distribuição das notas.

Tabela 1. Pré-teste vs. Pós-teste

\begin{tabular}{|l|c|c|c|c|c|c|}
\hline & $\begin{array}{c}\text { Total de } \\
\text { Pontos } \\
\text { Possíveis }\end{array}$ & $\begin{array}{c}\text { Total de } \\
\text { Pontos } \\
\text { Conquis- } \\
\text { tados }\end{array}$ & Média & Mediana & $\begin{array}{c}\text { Desvio } \\
\text { padrão }\end{array}$ & Variância \\
\hline Pré-teste & 280 & 194 & 6.93 & 7 & 1.25 & 1.55 \\
\hline Pós-teste & 280 & 221 & 7.89 & 8 & 1.42 & 2.03 \\
\hline
\end{tabular}


VIII Congresso Brasileiro de Informática na Educação (CBIE 2019)

Anais do XXX Simpósio Brasileiro de Informática na Educação (SBIE 2019)

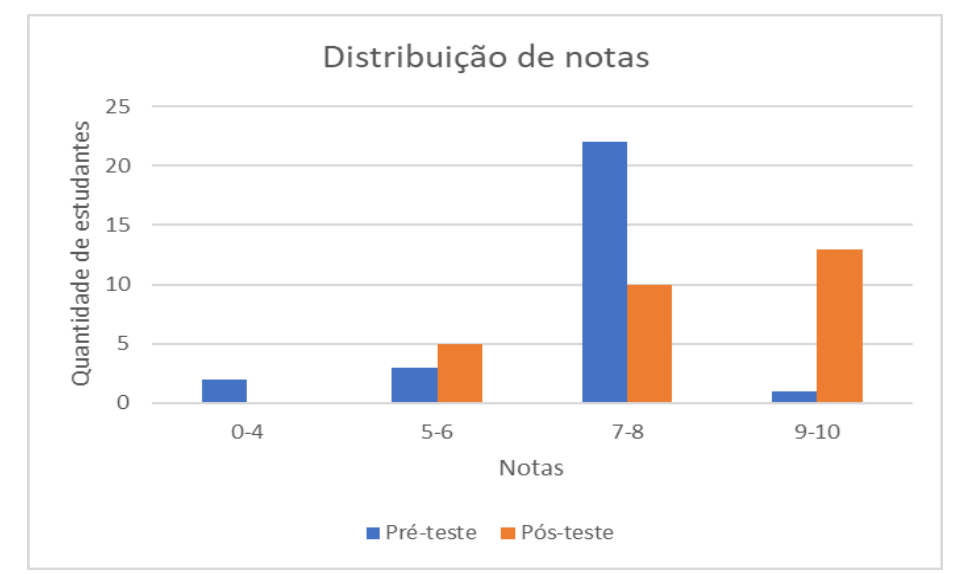

Figura 4 - Distribuição de notas entre pré-teste e pós-teste

Para testar a hipótese nula de que não houve diferença significativa entre as médias do pré e pós teste, foi executado um teste $t$ pareado resultando em $p$ valor $=0,005$, rejeitando a hipótese nula com nível de significância a $1 \%$. A diferença entre as médias foi aproximadamente $14 \%$.

\subsection{Competição}

O resultado da competição está apresentado na Tabela 2. Algumas equipes terminaram com pontuação negativa devido às respostas incorretas. A soma dos pontos positivos totalizou menos que 15 justamente porque alguns times perderam pontos. Durante a competição, os investigadores observaram um grande esforço pelos estudantes em tentarem ser o primeiro a levantar a mão com a resposta correta. Aconteceu inclusive uma mudança de estratégia: inicialmente levantavam a mão e respondiam sem a certeza. Com a evolução da competição, as equipes ficaram concentradas em abrir o modelo 3D e verificar a resposta.

Tabela 2. Resultados da competição

\begin{tabular}{|l|c|c|c|c|c|c|c|}
\hline Equipe & $\mathbf{1}$ & $\mathbf{2}$ & $\mathbf{3}$ & $\mathbf{4}$ & $\mathbf{5}$ & $\mathbf{6}$ & $\mathbf{7}$ \\
\hline Pontos & -1 & -1 & 2 & 1 & 3 & 0 & 4 \\
\hline
\end{tabular}

\subsection{Opinião dos estudantes}

A Tabela 3 apresenta as respostas das opiniões dos 28 estudantes. Observa-se que as quatro primeiras questões essencialmente foram respondidas positivamente, o que leva a considerar que os estudantes aprovaram o uso do aplicativo. A quinta questão permitia que os estudantes sugerissem alguma melhoria no aplicativo. As respostas a respeito do desempenho são relativas a alguns celulares que não atendiam o requisito mínimo de processamento exigido pelo Unity+Vuforia: ARM Cortex que implementa a arquitetura Armv7 com Neon ou Atom usando OpenGL ES 2.0. Quanto ao zoom, foram respostas de estudantes que gostariam de uma maior aproximação de visualização dos modelos 3D. Alguns estudantes sugeriram adicionar animações, pois o mapa continha somente elementos estáticos. E ainda, houveram sugestões para versão em iOS.

A última questão abria a possibilidade para que os estudantes expusessem as dificuldades enfrentadas com o aplicativo. O problema com o foco da câmera dizia 
VIII Congresso Brasileiro de Informática na Educação (CBIE 2019)

Anais do XXX Simpósio Brasileiro de Informática na Educação (SBIE 2019)

respeito a nitidez da qualidade da imagem capturada. Uma vez que o aplicativo não conseguia reconhecer a imagem de marcação, ele acabava não reproduzindo o modelo 3D. Esse é mais um problema em relação à limitação de hardware. Por essa razão, a maioria dos trabalhos relatados na seção 2, utilizaram marcadores de 2 cores (preto e branco). Porém, esses marcadores foram inseridos (colados) nos livros. O último problema relatado referia-se à instalação do aplicativo. Os investigadores forneceram uma URL encurtada para baixar o aplicativo. Porém, como a internet da escola não tinha um bom desempenho, acabou demorando um pouco mais para baixar o aplicativo.

Tabela 3. Resultados do questionário de opinião

\begin{tabular}{|l|l|}
\hline Questões & Respostas \\
\hline $\begin{array}{l}\text { 1. Você achou o aplicativo intuitivo e fácil de usar? } \\
\text { 2. O uso do aplicativo despertou teu interesse nos } \\
\text { conteúdos relacionados aos aspectos do continente } \\
\text { africano? }\end{array}$ & Sim: $96.4 \%$ Não: $3.6 \%$ \\
\hline $\begin{array}{l}\text { 3. Você acredita que com essa abordagem de } \\
\text { apresentação de conteúdos relacionados a aspectos } \\
\text { naturais da África, pôde ter lhe ajudado a entender } \\
\text { melhor os aspectos relacionados ao tópico? } 14.3 \%\end{array}$ & Sim: $96.4 \%$ Não: $3.6 \%$ \\
\hline $\begin{array}{l}\text { 4. Qual a sua avaliação sobre a utilidade do aplicativo? } \\
\text { S. Você pode sugerir alguma melhoria para o } \\
\text { aplicativo. }\end{array}$ & $\begin{array}{l}\text { Muito bom: } 28.6 \% \text { Bom: } 57.1 \% \text { Regular: } \\
10.7 \% \text { Não respondeu: } 3.6 \%\end{array}$ \\
\hline $\begin{array}{l}\text { Desempenho: } 16,7 \% \\
\text { Mais zoom: } 16,7 \% \\
\text { Adicionar animações: } 10 \% \\
\text { Versão para iOS: } 10 \% \\
\text { Não respondeu: } 46,6 \%\end{array}$ \\
\hline 6. Qual foi sua maior dificuldade em usar o aplicativo? & $\begin{array}{l}\text { Foco da câmera: } 30 \% \\
\text { Desempenho: } 13,3 \% \\
\text { Instalação: } 3,3 \% \\
\text { Não respondeu: } 53,4 \%\end{array}$ \\
\hline
\end{tabular}

\section{Discussões e Conclusões}

Esse trabalho apresentou o desenvolvimento de um aplicativo simples para dispositivos móveis com a capacidade de apresentar modelos 3D sobre os livros didáticos que os alunos já vinham utilizando. A melhoria no desempenho com a utilização do aplicativo foi mensurada usando testes aplicados antes e depois da experiência. Os resultados apresentaram uma diferença estatisticamente significativa entre ambas com uma melhoria de aproximadamente $14 \%$ no resultado dos testes. Adicionalmente, foram coletados algumas informações e opiniões sobre a satisfação com a experiência: $85,7 \%$ responderam que foi muito boa ou boa.

A experiência apresentada nesse trabalho demonstrou o interesse dos alunos em utilizar aplicativos em dispositivos móveis integrado ao livro didático, aumentando a relevância no assunto e tornando a aprendizagem mais significativa. Os investigadores acreditam que isso apenas seria o suficiente para motivar o investimento na produção desse tipo de aplicativo. O tempo de desenvolvimento foi curto pois a implementação do aplicativo é muito simples, onde o único esforço foi desenhar o modelo 3D no Blender, o que implica em um baixo custo de investimento. Não há necessidade de 
VIII Congresso Brasileiro de Informática na Educação (CBIE 2019)

Anais do XXX Simpósio Brasileiro de Informática na Educação (SBIE 2019)

trocar os livros didáticos, apenas investir em material adicional, através do desenvolvimento de aplicativos com RA.

\section{Agradecimentos}

Os autores agradecem à Profa. Sirlene França e os seus alunos do nono ano da disciplina de geografia da E.M. Christa Sedlacek por terem aceito participar da experiência e testado o aplicativo.

\section{Referências}

ADAMS, M.; ADAMS, S. Expedições Geográficas. 90 Ano. 2nd. ed. [s.1.] Editora Moderna, 2016.

AMIN, D.; GOVILKAR, S. Comparative Study of Augmented Reality SDK's. International Journal on Computational Science \& Applications, v. 5, n. 1, p. 11-26, 2015.

ASAI, K.; KOBAYASHI, H.; KONDO, T. Augmented Instructions - A Fusion of Augmented Reality and Printed Learning Materials. IEEE International Conference on Advanced Learning Technologies. Anais...2005.

BILLINGHURST, M.; DÜNSER, A. Augmented Reality in the Classroom. Computer, p. 5663, 2012.

BLENDER. About - blender.org. 2019. Disponível em: <https://www.blender.org/about/>. Acesso em: 18 maio. 2019.

BOWER, M. et al. Augmented Reality in education - cases, places and potentials. Educational Media International, v. 51, n. 1, p. 1-15, 2014.

CARDOSO, A. et al. Tecnologias para o desenvolvimento de sistemas de realidade virtual e aumentada. In: EDITORA UNIVERSITÁRIA UFPE (Ed.). . Tecnologia para o Desenvolvimento de Sistemas de Realidade Virtual e Aumentada. [s.l: s.n.]. p. 1-19.

CARDOSO, R. G. S. et al. Uso da realidade aumentada em auxílio à educação. Computer on the Beach. Anais...2014.

CHEN, S.; ZHANG, J. The Adaptive Learning System Based on Learning Style and Cognitive State. International Symposium on Knowledge Acquisition and Modeling. Anais...Ieee, dez. 2008Disponível em: $<$ http://ieeexplore.ieee.org/lpdocs/epic03/wrapper.htm?arnumber $=4732834>$. Acesso em: 18 jun. 2014.

CUENDET, S. et al. Designing augmented reality for the classroom. Computers \& Education, v. 68, p. 557-569, 2013.

GAZCÓN, N. F.; LARREGUI, J. I.; CASTRO, S. M. La Realidad Aumentada como Libros Aumentados y Reconstrucción 3D. Revista Iberoamericana de Educación en Tecnología y Tecnología en Educación, n. 17, p. 7-15, 2016.

GOMES, J. D. C.; GOMES, C. M. C.; OLIVEIRA, L. Realidade aumentada em ambientes formais de aprendizagem. 12th Iberian Conference on Information Systems and Technologies. Anais...2017.

GRASSET, R.; DÜNSER, A.; BILLINGHURST, M. Edutainment with a Mixed Reality Book: A visually augmented illustrative childrens , book. International Conference on Advances in Computer Entertainment Technology. Anais...2008. 
VIII Congresso Brasileiro de Informática na Educação (CBIE 2019)

Anais do XXX Simpósio Brasileiro de Informática na Educação (SBIE 2019)

GUTIÉRREZ, J. M.; ASME, M. C. Augmented Books Applied to Engineering: An Attractive Tool for the Student and Useful for Learning. International Design Engineering Technical Conferences and Computers and Information in Engineering Conference. Anais...2011.

HORNECKER, E.; DÜNSER, A. Supporting Early Literacy with Augmented Books Experiences with an Exploratory Study. GI Jahrestagung, v. 1, p. 555-559, 2007.

IVANOVA, G.; ALIEV, Y.; IVANOV, A. Augmented Reality Textbook for Future Blended Education. International Conference on e-Learning. Anais...2014.

JOHNSON, L. et al. Simple augmented realityThe 2010 Horizon Report. [s.l: s.n.].

KIRNER, T. G.; REIS, F. M. V.; KIRNER, C. Development of an Interactive Book with Augmented Reality for Teaching and Learning Geometric Shapes. 7th Iberian Conference on Information Systems and Technologies. Anais...2012.

MANN, S. et al. All Reality: Virtual, Augmented, Mixed (X), Mediated (X,Y), and Multimediated RealityarXiv:1804.08386, 2018. Disponível em: $<$ http://arxiv.org/abs/1804.08386>

MARTÍN-GUTIÉRREZ, J.; CONTERO, M.; ALCAÑIZ, M. Evaluating the Usability of an Augmented Reality Based Educational Application. International Conference on Intelligent Tutoring Systems. Anais...2010.

NETO, J. M.; FRACALANZA, H. O Livro Didático de Ciências: problemas e soluções. Ciência \& Educação, v. 9, n. 2, p. 147-157, 2003.

PTC. Industrial Augmented Reality. 2019. Disponível em: $<$ https://www.ptc.com/en/products/augmented-reality>. Acesso em: 18 maio. 2019.

REGUERAS, L. M. et al. Effects of Competitive E-Learning Tools on Higher Education Students : A Case Study. IEEE Transactions on Education, v. 52, n. 2, p. 279-285, 2009.

SCHMITZ, E. M.; SOLANO, D.; LOPES, M. C. Desenvolvimento de um aplicativo para auxiliar no ensino de sistema solar utilizando realidade aumentada. Revista de Sistemas e Computação, v. 7, n. 2, p. 337-352, 2017.

UNITY. Unity - System Requirements. 2019a. Disponível em: $<$ https://unity3d.com/pt/unity/system-requirements>. Acesso em: 18 maio. 2019.

UNITY. Unity Public Relations. 2019b. Disponível em: <https://unity3d.com/pt/publicrelations>. Acesso em: 18 maio. 2019.

YANG, H. S. et al. Hybrid visual tracking for augmented books. International Conference on Entertainment Computing. Anais...Springer Berlin Heidelberg, 2008.

YUEN, S. C.-Y.; YAOYUNEYONG, G.; JOHNSON, E. Augmented Reality: An Overview and Five Directions for AR in Education Augmented Reality. Journal of Educational Technology Development and Exchange, v. 4, n. 1, p. 119-140, 2011. 